\title{
Laboreal
}

Volume $8 \mathrm{~N}^{\circ} 1$ | 2012

Género, Atividades e Saúde

\section{Análisis de algunos mecanismos de adquisición de los primeros elementos de un oficio en el adolescente}

Análise de alguns mecanismos de aquisição dos primeiros elementos de uma profissão pelo adolescente

Analyse de quelques mécanismes d'acquisition des premiers éléments d'un métier chez l'adolescent

Analysis of some mechanisms the teenager uses to acquire the first elements of a profession

\section{Antoine Léon}

\section{(2) OpenEdition}

\section{Journals}

Edición electrónica

URL: http://journals.openedition.org/laboreal/7401

DOI: 10.4000/laboreal.7401

ISSN: 1646-5237

Editor

Universidade do Porto

Referencia electrónica

Antoine Léon, «Análisis de algunos mecanismos de adquisición de los primeros elementos de un oficio en el adolescente », Laboreal [En línea], Volume 8 Nº1 | 2012, Publicado el 01 diciembre 2012, consultado el 10 diciembre 2020. URL : http://journals.openedition.org/laboreal/7401 ; DOI : https:// doi.org/10.4000/laboreal.7401

Este documento fue generado automáticamente el 10 diciembre 2020.

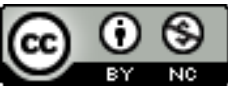

Laboreal está licenciado com uma Licença Creative Commons - Atribuição-NãoComercial 4.0 Internacional. 


\section{Análisis de algunos mecanismos de adquisición de los primeros elementos de un oficio en el adolescente}

Análise de alguns mecanismos de aquisição dos primeiros elementos de uma profissão pelo adolescente

Analyse de quelques mécanismes d'acquisition des premiers éléments d'un métier chez l'adolescent

Analysis of some mechanisms the teenager uses to acquire the first elements of a profession

\section{Antoine Léon}

\section{REFERENCIA}

Artículo original : Léon, A. (1957). Analyse de quelques mécanismes d'acquisition des premiers éléments d'un métier chez l'adolescent.

Bulletin du CERP, 2, 183-188.

1 Las observaciones que se van a presentar aquí son el resultado de un trabajo realizado en colaboración con C. Dacquin, profesor técnico adjunto en el Centro de aplicación de la Escuela normal nacional de aprendizaje de París y siguen la línea de las cuestiones definidas por S. Pacaud en el número especial del Journal de Psychologie dedicado a psicología laboral ( n. 1 de 1955) :

2 “... El fracaso de un individuo en su oficio no radica casi nunca en el hecho de que no ha podido aprender acciones correctas, sino en que éste no ha sabido desprenderse de las acciones no adecuadas. Dicho de otro modo, en la cadena de asociaciones que conducen ya sea a operaciones motrices o a operaciones intelectuales y mentales, además de las 
asociaciones seleccionadas por la formación profesional, se activan otras conexiones, primero fortuitas, después facilitadas por la repetición, y que determinan errores profesionales en los ejecutantes..."

3 ¿Cuál es el contenido de estos errores y cuáles son las condiciones concretas de la formación de estas conexiones defectuosas durante el aprendizaje de oficio en el adolescente?

4 Intentaremos aportar algunos elementos de respuesta a esta doble pregunta, a la vez psicológica y pedagógica, estudiando algunos de los mecanismos de adquisición de los rudimentos del oficio de fresador por parte de 25 alumnos de segundo curso de un centro de enseñanza pública.

\section{Método de observación y de control}

5 Los alumnos examinados son aprendices de 15-16 años que ya han cursado un primer año de ajuste y que, al empezar el segundo curso, han realizado prácticas de orientación y de iniciación en los tres talleres de mecánica (ajuste, torneado y fresado) antes de ser asignados al taller de su elección.

6 La iniciación al fresado abarca un periodo de una semana que incluye 18 horas presenciales efectivas en el taller. Los alumnos llegan en grupos de cuatro y trabajan en máquinas agrupadas en un espacio reducido. De este modo es posible proceder no sólo al estudio de las conductas individuales de los aprendices, sino también a comparaciones de conjunto mediante la alternancia de los grupos sometidos a medios educativos distintos.

$7 \quad 1^{\circ}$ Estudio de las conductas individuales. En la primera fase del estudio se ha elaborado el balance de las dificultades detectadas y de los errores cometidos por los aprendices durante las prácticas de iniciación. Se evalúa a los alumnos, ya sea directamente delante de la máquina, o durante la entrevista posterior a un ejercicio de control. De este modo puede lanzarse una primera hipótesis sobre el contenido de algunos errores observados: el mecanismo en cuestión aparece como un proceso de generalización precipitada. En una segunda etapa, situada en el inicio del curso escolar 1955-1956, a la luz de esta hipótesis -y teniendo en cuenta los medios pedagógicos de los que dispone el aprendiz- se analizan las conductas reales practicadas para afrontar las dificultades encontradas. Los alumnos son invitados a explicar, a justificar por sí mismos, una u otra práctica profesional particularmente difícil. En ocasiones, se crean situacionesproblema en la propia máquina para ilustrar mejor el proceso estudiado.

$8 \quad 2^{\circ}$ Comparaciones de conjunto. Para controlar la función que desempeñan algunos factores educativos en la génesis de los errores profesionales, hemos separado a los alumnos en dos conjuntos y los hemos sometido a dispositivos pedagógicos parcialmente distintos. Mientras que los alumnos del grupo "testigo" se inician en el fresado siguiendo un método que podríamos calificar, con reservas, como global o intuitivo, a los del grupo "experimental" se les invita constantemente a practicar un esfuerzo de análisis y de uso de conocimientos generales en la fase preparatoria del trabajo propiamente dicho sobre la máquina.

9 La comparación de ambos gruposse realiza con un ejercicio de evaluación final que incluye, en una situación nueva, problemas idénticos a los realizados durante las prácticas. Tras el examen de evaluación, los aprendices se someten a una entrevista 
oral individual durante la cual se les plantean pruebas diseñadas sobre el modelo de las principales dificultades encontradas durante el aprendizaje.

10 No entraremos aquí a examinar todas las dificultades. Nos limitaremos a algunas observaciones psicológicas relativas a la asimilación de las primeras reglas del fresado.

11 Ante la imposibilidad de poder aportar ejemplos concretos cuya relación exigiría desarrollos tecnológicos, presentaremos bajo una forma elaborada el contenido y las condiciones educativas de algunos errores profesionales cometidos por los aprendices de fresador.

\section{De la generalización prematura a la generalización correcta en la adquisición de las primeras reglas del fresado}

Durante nuestras observaciones, pudimos constatar paulatinamente el fenómeno siguiente : entre las explicaciones y las instrucciones orales o escritas facilitadas por el profesor técnico y las respuestas efectivas (verbales o motrices) del aprendiz, se intercala una actividad más o menos consciente que consiste en que el aprendiz o bien generaliza algunos aspectos parciales de dichas explicaciones e instrucciones, o bien crea conexiones a espaldas del profesor durante el trabajo personal del alumno. Analizaremos este fenómeno y a continuación precisaremos algunas de sus condiciones.

1ํA Algunos aspectos del proceso de generalización.

Estos se presentan bajo distintas formas.

a) En ocasiones son el fruto de un análisis previo insuficiente y consisten en la transposición mecánica de una situación A a una situación B de exigencias secundarias o particulares de la primera situación. Así, la aplicación de una determinada regla, válida para un ejercicio determinado, se extiende equivocadamente al resto de ejercicios. Esta regla es separada de su contexto, es decir, del conjunto de exigencias de la tarea. Un aprendiz manipula tal volante para ajustar la máquina en un ejercicio determinado y sigue utilizando el mismo volante cuando las condiciones de trabajo han cambiado.

16 b) En otros casos, los procesos de generalización son el resultado de un análisis previo y se presentan como una extrapolación de relaciones más o menos complejas y percibidas de un modo más o menos correcto. Así, los aprendices extrapolan a situaciones nuevas relaciones espaciotemporales percibidas entre las operaciones sucesivas de un ejercicio determinado. Estos descubren, por ejemplo, algunas relaciones de simetría en las posiciones que ocupa la herramienta en ejercicios sucesivos.

17 Los aprendices generalizan relaciones para determinar la posición de la herramienta en un ejercicio nuevo.

18 A menudo, trasponen el orden de las operaciones realizadas durante las prácticas en el desarrollo de las operaciones del ejercicio de evaluación. En ocasiones justifican la existencia de las relaciones percibidas argumentando lo que creen que son las intenciones del profesor técnico. "En el primer ejercicio, declaran estos en suma, nos han pedido que ajustásemos la máquina con este volante, en el segundo ejercicio, con éste otro, por tanto, en el tercer ejercicio, utilizaremos este último volante para conocer bien la máquina." 
19 Encontramos este fenómeno de interpretación de las intenciones pedagógicas del profesor en el modo en que los aprendices utilizan las instrucciones técnicas que completan el dibujo de la pieza que se va a realizar. Si hay veces en las que las instrucciones precisas de ejecución dispensan al alumno de cualquier esfuerzo de búsqueda, en ocasiones éstas le conducen a realizar comparaciones entre los ejercicios sucesivos de una progresión. En lugar de preparar su trabajo en función de las exigencias precisas de la tarea actual, se limita a interpretar algunos cambios producidos en las condiciones de dicha tarea haciendo intervenir lo que él cree que son las intenciones pedagógicas de su profesor.

La toma de conciencia de los distintos tipos de relaciones que acabamos de plantear da lugar a la elaboración, por parte de los propios alumnos, de auténticas reglas que sustituyen a las instrucciones facilitadas. Eficaz en algunos casos, la aplicación de estas "reglas" conduce, en algunas circunstancias, a soluciones erróneas.

c) El ejemplo más impactante del proceso de generalización estudiado nos viene dada por la regla "descubierta" individualmente y aplicada por todos los aprendices observados para proceder a la operación más importante del ajuste de la máquina cuando menos en el nivel de iniciación al oficio. Este fenómeno apareció con un tal relieve, con un grado tal de generalización, mejor aún, la regla "descubierta" por los alumnos era expresada con una homogeneidad tal, que debemos subrayar algunas observaciones relacionadas. Esta regla, prácticamente válida para muchos casos, refleja sólo la secuencia de los movimientos ejecutados realmente en estos casos (giro del volante de la máquina en determinado sentido y después en sentido opuesto).

¿Cómo se explica la sustitución de una regla de este tipo a la enseñada por el profesor técnico?

$23 \mathrm{Si}$, durante su trabajo, el alumno recurre a una regla simple resultante de la toma de conciencia de relaciones motrices que se crean y se consolidan durante la actividad práctica, es sin duda porque se ve repelido por el número y la complejidad de las operaciones intelectuales que exige la aplicación de las enseñanzas del profesor especialmente la aplicación de las leyes de composición de las fuerzas. Debido a la persistencia y a la solidez de estas relaciones, la mayoría de aprendices, al enfrentarse a una tarea en la que la regla "descubierta" por su cuenta se revela manifiestamente inaplicable, son incapaces de aplicar la regla explicada por el educador. No obstante, algunos de ellos, minutos antes han resuelto correctamente en un esquema el problema planteado.

24 Así, la aplicación correcta de las reglas en un trabajo esquemático no garantiza su asimilación por parte de los alumnos. El paso del ejercicio teórico con esquemas resultado de las acciones educativas "intencionales" ejercidas por el profesor técnicoal acto motor propiamente dicho no es simple, mecánico. Nuevos condicionantes, que son fruto de la actividad propia del alumno, influyen en este paso haciendo intervenir elementos formativos que podrían calificarse de "no intencionales" porque se manifiestan a espaldas del educador. Estas dos formas de condicionamiento están más o menos bien integradas en el mismo alumno.

En ocasiones ocurre que un aprendiz, tras haber "repetido" correctamente las reglas enseñadas por el profesor técnico, cuando se trata de manipular la máquina, pasa a las pseudorreglas que se ha creado él mismo. Con este aprendiz, se podrían invertir los 
términos del siguiente aforismo según el cual "el hombre de hoy piensa como Copérnico y habla como Ptolomeo" cuando declara que "el sol sale o se pone".

A veces, el alumno pasa con facilidad de un modo de explicación a otro, basándose tanto en elementos conceptuales o representativos de la demostración del profesor, como en los datos de su propia experiencia, en apreciaciones que proceden de una visión global, intuitiva, del mecanizado de la pieza.

A la luz de estas observaciones, uno de los principales problemas que se plantean a la vez el psicólogo y el educador es saber si la intervención de determinados factores educativos "intencionales" puede contribuir a mejorar el proceso de generalización del aprendiz, es decir, en definitiva, a reducir la importancia de los errores profesionales.

Tabla 1

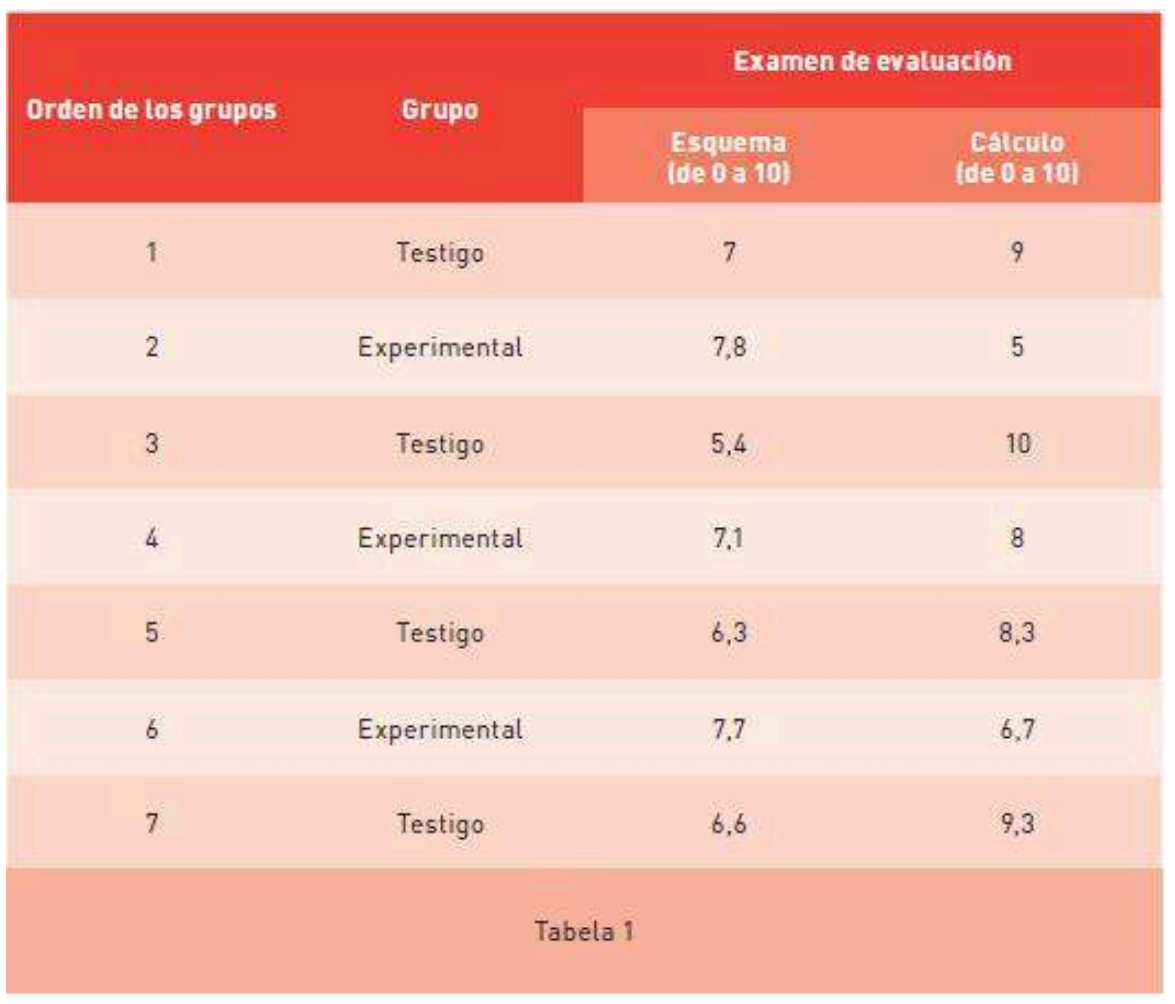

$2^{2}$ Medios educativos y procesos de generalización. La comparación de los resultados obtenidos en el examen de evaluación por dos conjuntos de alumnos sometidos a dispositivos pedagógicos distintos podría, en cierta medida, aportar una respuesta a este problema. Este examen de evaluación consta, por una parte, de un ejercicio de aplicación, en un esquema, de las reglas de fresado y, por otra, de un ejercicio de cálculo sobre el funcionamiento de la máquina. Sin duda este tipo de pruebas tienen un alcance limitado, porque lo que reproducen no es un trabajo efectivo en la máquina, sino sólo la fase preparatoria de dicho trabajo. Éstas constituyen, no obstante, el soporte de algunas de las generalizaciones estudiadas anteriormente.

El ejercicio del esquema ofrece una ventaja apreciable al conjunto experimental (conjunto que ha sido incitado a realizar un esfuerzo de análisis). Éste último obtiene una media de 7,5 sobre 10 contra un 6,3 del conjunto testigo. Pero esta clasificación se invierte cuando se usa como base comparativa el ejercicio de cálculo : 6,5 sobre 10 para el primer conjunto contra 9,2 para el segundo. Sin duda, estos números deben 
interpretarse con cautela dado el bajo número de individuos participantes (11 alumnos para el primer conjunto, 14 para el segundo) y por otra parte, por el no emparejamiento sistemático de los dos conjuntos.

No obstante, debemos darle un cierto valor si se tiene en cuenta, por una parte, la discordancia de los resultados obtenidos en los dos ejercicios y, por otra parte, la persistencia de esta discordancia en los resultados calculados, no globalmente, sino grupo por grupo. Los resultados, clasificados por orden cronológico [1], obtenidos por los siete grupos que hicieron las prácticas de fresado se recogen en la tabla I. Para explicar esta serie de discordancias, creemos que es conveniente remitirse a las condiciones precisas en las que se han desarrollados las prácticas para los dos grupos. En la preparación del trabajo, la actividad de investigación y de análisis se practicó sobre todo con los alumnos del conjunto experimental. Se invitó a éstos últimos a distinguir, en particular en la fase de preparación del trabajo, las distintas fuerzas resultantes del contacto de la herramienta y de la pieza. Esta actividad parece haber mejorado la calidad del proceso de generalización, es decir, en definitiva, la calidad de las respuestas dadas por el alumno en la situación nueva del examen. De hecho, tenemos razones para pensar que el análisis en profundidad de una situación permite distinguir los aspectos secundarios de los aspectos esenciales de esta situación y operar generalizaciones correctas a partir de estas últimas. Así pues, se apunta la superioridad relativa del conjunto experimental sobre el grupo testigo en el ejercicio del esquema.

31 Si ahora ya no consideramos los resultados de este ejercicio sino los de la prueba de cálculo, los alumnos del grupo testigo se revelan claramente superiores a los del conjunto experimental. Aquí también es preciso tener en cuenta la actividad real desarrollada por los aprendices durante las prácticas. Los alumnos del grupo experimental disponían, entre otros medios educativos, de hojas de cálculo mimeografiado con las fórmulas y los significados de los símbolos. Su trabajo consistía en aplicar correctamente estas fórmulas ya aprendidas en el primer año de aprendizaje. Estos alumnos se encontraban exentos de cualquier esfuerzo de evocación o de comprensión. En cambio, los aprendices del grupo testigo estaban obligados a hacer dicho esfuerzo. Algunos de ellos incluso han afirmado que habían sido incitados a repasar su cuaderno de tecnología de primer año. Ello explica que hubieran podido tener mejores resultados que sus compañeros en el ejercicio de cálculo.

Si las constataciones que se acaban de hacer no pueden, como ocurre en cualquier examen de evaluación final, dar cuenta de manera suficiente de los mecanismos de asimilación de los conocimientos académicos o profesionales, sí que contribuyen a sostener algunas prácticas pedagógicas, a arrojar luz sobre algunos medios educativos "intencionales".

Ahora trataremos los aspectos pedagógicos que se han desarrollado aparte para concluir con las observaciones más generales.

\section{Conclusiones}

En lo que se refiere a la asimilación de las nociones básicas del fresado, hemos analizado el contenido de una actividad que, suscitada por el profesor técnico, después se desarrolla un poco a espaldas de éste en forma de generalizaciones diversas y en función de elementos educativos "no intencionales". 
35 En realidad, los elementos se sitúan en planos distintos. Algunos pertenecen a un entorno educativo limitado e inmediato: relaciones espacio-temporales elaboradas a partir de indicios visuales o motores que extraen por sí mismos su significado del contacto directo del adolescente con la máquina. Otros tienen que ver con un entorno educativo más amplio: el aprendiz utiliza más o menos bien algunos conocimientos enseñados por el profesor de ciencias (leyes de la composición de las fuerzas). Por último, otros se refieren a toda la experiencia escolar del aprendiz: la noción de "progresión" de los ejercicios, forjada a lo largo de su vida escolar, le lleva a interpretar las intenciones pedagógicas actuales de su profesor técnico.

Sobra decir que un estudio más completo de las condiciones de aprendizaje del oficio debería incluir otros factores como por ejemplo la motivación con todo su conjunto de determinaciones sociales.

Las observaciones psicológicas que se acaban de presentar sobre la asimilación de las primeras reglas del fresado son en parte generalizables y confirman, por ejemplo, algunas tesis formuladas por Ombredane y Faverge en su obra reciente dedicada al análisis del trabajo. Tras haber subrayado la importancia de la función que desempeñan las señales (tonos del metal caliente, indicaciones del manómetro) en el desempeño de los actos profesionales, estos autores ilustran con numerosos ejemplos los aspectos que en una tarea determinada "son imprevistos y están sujetos al descubrimiento del trabajador."

38 Al examinar de cerca el mecanismo de la orientación profesional o, más precisamente, el proceso de elaboración de la elección profesional por parte del niño, también descubrimos el desarrollo de una actividad "autónoma" que en cierto modo escapa al control del maestro o al del consejero de orientación profesional. Así, la información sobre algunas familias de oficios crea en los alumnos del último año de primaria, una actitud -hecha de una mayor claridad en la elección o el rechazo de profesiones- que tiende a generalizarse durante la cual el niño elabora los materiales diversos procedentes de su entorno, en particular de su familia [2].

Si abandonamos el ámbito de las profesiones para centrarnos en el las disciplinas académicas, se observan mecanismos intelectuales comparables, en cierto modo, a los que hemos descrito aquí. Así, J. Levine [3], basándose en algunas tesis de Wallon, demuestra que la actividad de adquisición "consiste en elaborar una especie de copia del texto y que esa copia, superpuesta o incluso sustituida en algunos casos por las trazas directas que deja la audición, constituye la materia fundamental de la memoria". La actividad de identificación de las realidades expuestas en un texto escapa, en gran medida, a la influencia directa del educador debido a que la experiencia social extraescolar del niño entra aquí de forma considerable.

Estas pocas relaciones, que merecen un mayor desarrollo, contribuyen no obstante a confirmar el punto de vista según el cual los problemas psicológicos y pedagógicos presentados por la iniciación a la profesión no son esencialmente distintos de los que plantea la enseñanza de las materias denominadas generales.

41 Por último, a propósito de este estudio, quisiéramos evocar un problema más general.

42 ¿Los hechos que se acaban de analizar competen a la psicología o a la pedagogía? Parece que pertenecen a la vez a ambas disciplinas. No sólo se refieren a las condiciones externas de la asimilación de un sector determinado de conocimientos - la enseñanza profesional- sino también a los mecanismos internos de dicha asimilación. ¿Podemos 
hablar de confusión entre ámbitos supuestamente distintos o de degradación de la psicología con el pretexto de que las observaciones tienen por objeto la actividad escolar y conducen a algunas aplicaciones pedagógicas? Creemos que no. La diversidad de las dependencias del hecho psíquico legitima tanto la investigación psicopedagógica como la investigación psicofisiológica, la investigación en laboratorio como la relativa a la actividad normal -por ejemplo escolar o profesional- del hombre. Dicho en otros términos, la psicopedagogía, lejos de ser una categoría cómoda pensada para agrupar los fenómenos mal definidos, responde por lo contrario a la propia naturaleza de algunos hechos que engloba la psicología.

Más importantes que las distinciones que nos esforzamos por hacer entre los campos de actividad que se solapan necesariamente sin que por ello se confundan, nos parecen ser las divergencias entre los métodos, entre las vías de acceso a estos métodos.

[1] Hemos alternado los grupos del conjunto experimental y los del conjunto testigo para igualar, en estos dos conjuntos, la posible influencia de las prácticas anteriores. De hecho, podemos suponer que los aprendices del séptimo grupo que hicieron las prácticas a finales de noviembre (después de haber pasado por ajuste y torneado) puedan obtener mejores resultados que los del primer grupo que hacen las prácticas a principios de curso en octubre.

[3] J. LEVINE : Aspects élémentaires de l'assimilation d'un enseignement vital. Enfance, 1955 p. 8, 119-167.

\section{NOTAS}

1. Hemos alternado los grupos del conjunto experimental y los del conjunto testigo para igualar, en estos dos conjuntos, la posible influencia de las prácticas anteriores. De hecho, podemos suponer que los aprendices del séptimo grupo que hicieron las prácticas a finales de noviembre (después de haber pasado por ajuste y torneado) puedan obtener mejores resultados que los del primer grupo que hacen las prácticas a principios de curso en octubre.

2. B. I. N. O. P no 1 de 1954 y no 3 de 1955.

3. J. LEVINE: Aspects élémentaires de l'assimilation d'un enseignement vital. Enfance, 1955 p. 8, 119-167.

\section{RESÚMENES}

Toda falta profesional comporta a la vez un aspecto negativo y otro positivo ya que el individuo ha sustituido en su trabajo la respuesta correcta por la respuesta inadecuada. Esta observación 
psicopedagógica corriente es objeto de un análisis consagrado al comportamiento en su oficio de los aprendices de fresador (máquinas de fresar). Sucesivamente se expone el método de observación y de control y luego los resultados que ponen al descubierto la importancia del fenómeno de generalización en la génesis de las faltas profesionales.

Todo o erro profissional tem ao mesmo tempo um aspecto negativo e outro positivo. No seu trabalho, o indivíduo substitui uma resposta inadequada pela resposta correcta. É esta observação psicopedagógica corrente que é alvo de uma análise dedicada ao comportamento dos aprendizes fresadores. Expõe-se sucessivamente o método de observação e de controlo, e, de seguida, os resultados que evidenciam a importância dos fenómenos de generalização na génese dos erros profissionais.

Toute faute professionnelle comporte à la fois un aspect négatif et un autre positif, l'individu substituant dans son travail une réponse inadéquate à la réponse correcte. C'est cette observation psychopédagogique courant qui fait l'objet d'une analyse, consacrée au comportement des apprentis fraiseurs. On expose successivement la méthode d'observation et de contrôle, puis les résultats qui mettent en lumière l'importance du phénomène de généralisation dans la genèse des fautes professionnelles.

Every professional fault includes at one and the same time a positive and negative aspect, for the reason that in his work the individual substitutes an inadequate response for the correct one. This current psychopedagogic observation forms the subject of an analysis devoted to the behavior of the apprentices working milling machines. The method of observation and checking in first set forth and then the results which throw light on the importance of the phenomenum of generalization in the origin of professional faults.

\section{AUTOR}

\section{ANTOINE LÉON}

Service de Recherche de l'Institut National d'Etude du Travail et d'Orientation Professionnelle (INETOP) 